\title{
The Motion of the OH Group in p-Chlorophenol and its Influence on the ${ }^{35} \mathrm{Cl}$ NQR Parameters*, **
}

\author{
Mariano J. Zuriaga ${ }^{1}$ and Carlos A. Martín ${ }^{2}$ \\ Facultad de Matemática, Astronomía y Física (IMAF), Universidad Nacional de Córdoba, \\ Laprida 854, 5000 Córdoba, Argentina
}

Z. Naturforsch. 41 a, 408-411 (1986); received July 22, 1985

The ${ }^{35} \mathrm{Cl} \mathrm{NQR}$ transition frequencies and the spin-lattice relaxation times, $T_{1}$, for both lines in p-chlorophenol have been measured in the temperature range $90-310 \mathrm{~K}$. The frequency difference and the temperature derivatives for both lines clearly show the existence of two temperature intervals with distinct lattice contributions to the EFG. Similarly, $T_{1}$ data show a normal behaviour due to spin-phonon interactions up to $240 \mathrm{~K}$. Above this temperature $T_{1}$ begins to shorten in an exponential manner. The hindered motions of the $\mathrm{OH}$ group are proposed as responsibles of these effects, and an activation energy of $26 \mathrm{~kJ} \mathrm{~mol}^{-1}$ is determined.

\section{Introduction}

Crystalline p-chlorophenol in the stable $\alpha$ phase has two ${ }^{35} \mathrm{Cl}$ nuclear quadrupole resonances (NQR) [1] due to the existence of two crystallographically non-equivalent chlorine sites. The temperature dependence of both resonances looks normal, and the splitting between the two lines exhibits a slight change over a wide temperature range. Previous Zeeman effect studies $[2,3]$ reveal that the higher frequency line $v^{+}$corresponds to resonant nuclei located such that the $\mathrm{Cl}-\mathrm{O}$ vector makes a small angle with the $(010)$ plane (Type II molecules according to Perrin and Michel's notation, [4]), and the low frequency line $v^{-}$is associated with chlorines such that its $\mathrm{Cl}-\mathrm{O}$ vector makes a large angle with the (010) plane (Type I molecules). In this paper the temperature dependence of the NQR frequency, $v_{\mathrm{Q}}(T)$, for both resonance lines, and the spin-lattice relaxation time, $T_{1}(T)$, are reported and the possibility of hindered reorientational motion of the $\mathrm{OH}$ groups is examined.

\footnotetext{
* Presented at the VIIIth International Symposium on Nuclear Quadrupole Resonance Spectroscopy, Darmstadt, July $22-26,1985$.

** This research was partially supported by Consejo Nacional de Investigaciones Científicas y Técnicas (CONICET) and by Consejo de Investigaciones Científicas y Tecnológicas de la Provincia de Córdoba (CONICOR).

${ }^{1}$ Holder of a scholarship granted by CONICET.

${ }^{2}$ Fellow of the CONICET.
}

Reprint requests to Dr. C. A. Martín, Facultad de Matemática, Astronomía y Física (IMAF), Universidad Nacional de Córdoba, Laprida 854, 5000 Córdoba, Argentina.

\section{Experimental}

A powder p-chlorophenol sample, provided by Fluka (catalog number 25850), was used.

The ${ }^{35} \mathrm{Cl}$ NQR frequency was measured by means of a super-regenerative type spectrometer with an error of about $+/-300 \mathrm{~Hz}$. The spin-lattice relaxation time was measured using both continuous pulse (for $T_{1}>30 \mathrm{~ms}$ ) and $\pi / 2-\pi / 2$ pulse (for $\left.T_{1}<30 \mathrm{~ms}\right)$ sequences in a conventional pulsed spectrometer.

\section{Temperature Dependence of the NQR Frequency}

The ${ }^{35} \mathrm{Cl} \mathrm{NQR}$ frequency, $v_{\mathrm{Q}}(T)$, was measured in the temperature range 90 to $310 \mathrm{~K}$. The data are shown in Figure 1.

Figure 2 shows the frequency difference $\Delta v_{\mathrm{Q}}=$ $v^{+}-v^{-}$vs. $T$. A change in the curvature of $\Delta v_{\mathrm{Q}}$ is observed at about $240 \mathrm{~K}$, which is correlated to a marked change in the shape of $T_{1}(T)$, Fig. 4, for both lines. Therefore, the analysis of $v_{\mathrm{Q}}(T)$ is restricted to the low temperature range by means of Bayer's expression [5]

$$
v_{\mathrm{Q}}(T)=v_{0}\left[1-3 / 2\left\langle\theta^{2}\right\rangle\right],
$$

where $v_{0}$ is the static lattice resonance frequency. The asymmetry parameter has been neglected since its value was shown to be about $0.08(3) .\left\langle\theta^{2}\right\rangle$ is given by [6]

$$
\left\langle\theta^{2}\right\rangle=\frac{\hbar}{2} \sum_{j} \frac{1}{I_{j} \omega_{j}} \operatorname{coth}\left(\frac{\hbar \omega_{j}}{2 k T}\right),
$$




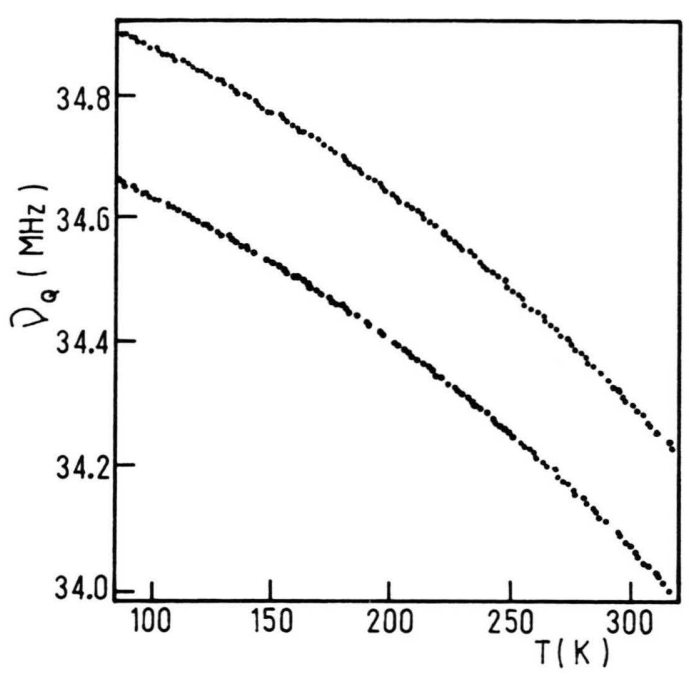

Fig. 1. Temperature dependence of the lower $\left(v^{-}\right)$and upper $\left(v^{+}\right) \mathrm{NQR}$ frequencies.

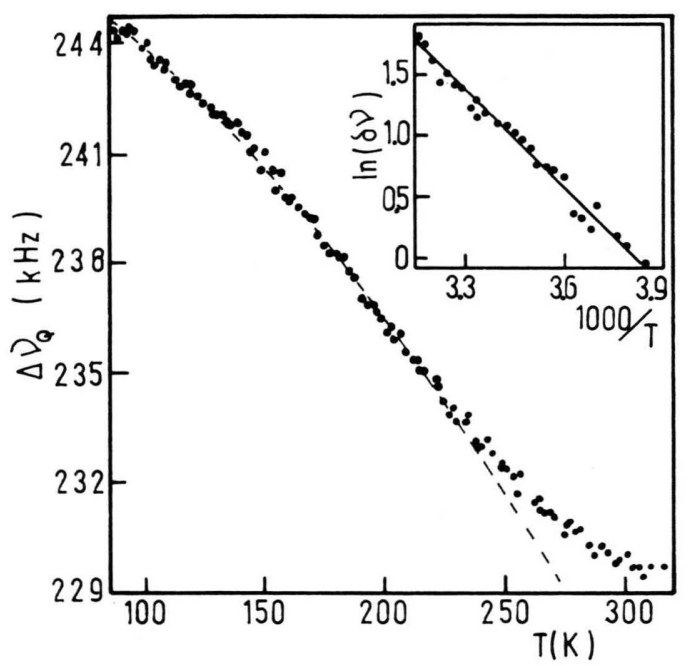

Fig. 2. Frequency difference between $v^{+}$and $v^{-}\left(\Delta v_{\mathrm{Q}}\right)$. Dashed line corresponds to a fit with a second degree polynomial. The insert shows the difference between the $\Delta v_{\mathrm{O}}$ data and the dashed line $(\delta v$ in $\mathrm{kHz})$ as a function of $1000 / T(T$ in $\mathrm{K})$.

where the sum is taken including all the internal bending of the $\mathrm{Cl}-\mathrm{C}$ bond and the torsional lattice modes. In order to evaluate the contribution of the internal modes to $\left\langle\theta^{2}\right\rangle$ we use the eigen-vectors and frequencies given by Varsanyi [7], and following
O'Leary [8] to calculate the $I_{j \text { 's }}$ we may write

$$
\begin{aligned}
\left\langle\theta^{2}\right\rangle= & 3.72 \cdot 10^{-4} \operatorname{coth}\left(\frac{312.2}{T}\right) \\
& +7.05 \cdot 10^{-4} \operatorname{coth}\left(\frac{238.1}{T}\right) \\
& +6.69 \cdot 10^{-4} \operatorname{coth}\left(\frac{118.0}{T}\right) \\
& +4.05 \cdot 10^{-4} \operatorname{coth}\left(\frac{195.0}{T}\right) \\
& +\frac{\hbar}{2 I_{\mathrm{e}} \omega_{\mathrm{e}}} \operatorname{coth}\left(\frac{\hbar \omega_{\mathrm{e}}}{2 k T}\right),
\end{aligned}
$$

where the four first terms are internal mode contributions and the last accounts for the lattice contribution. $\omega_{\mathrm{e}}$ is an effective lattice librational frequency related to $\omega_{x}$ and $\omega_{y}$ by

$\frac{1}{I_{\mathrm{e}}}=\frac{1}{I_{x}}+\frac{1}{I_{y}} ; \quad \frac{1}{I_{\mathrm{e}} \omega_{\mathrm{e}}^{2}}=\frac{1}{I_{x} \omega_{x}^{2}}+\frac{1}{I_{y} \omega_{y}^{2}}$

with $I_{x}, \omega_{x}$ and $I_{y}, \omega_{y}$ being the molecular moments of inertia and the librational frequencies about the $x$ and $y$ axes of the Electric Field Gradient (EFG), respectively. The $z$-axis is along the $\mathrm{Cl}-\mathrm{C}$ direction and the $x$-axis is perpendicular to the benzene ring.

In order to take into account thermal expansion effects on the lattice frequencies a linear temperature behaviour is adopted as proposed by Brown [9]:

$$
\omega_{\mathrm{e}}=\omega_{\mathrm{e} 0}\left(1-C_{\mathrm{e}} T\right) .
$$

Equations (3), (4) and (5) permit us to fit the frequency data for $T<240 \mathrm{~K}$. The following values are obtained:

$v_{0}^{+}=(35173.6 \pm 0.4) \mathrm{kHz}, \quad \omega_{\mathrm{e} 0}^{+}=(60.6 \pm 0.2) \mathrm{cm}^{-1}$, $C_{\mathrm{e}}^{+}=(8.08 \pm 0.04) 10^{-4} \mathrm{~K}^{-1}$,

$v_{0}^{-}=(34923.3 \pm 0.4) \mathrm{kHz}, \quad \omega_{\mathrm{e} 0}^{-}=(61.6 \pm 0.2) \mathrm{cm}^{-1}$

and $C_{\mathrm{e}}^{-}=(8.13 \pm 0.04) 10^{-4} \mathrm{~K}^{-1}$,

Note that the temperature dependences of the effective frequency, $\omega_{\mathrm{e}}$, obtained for both $v^{+}$and $v^{-}$ are similar. Fitting the frequency data in the whole temperature range yields effective torsional frequencies differing by about $10 \%$ for the two lines.

The obtained effective torsional frequencies are in agreement with previous measurements [10] and the temperature coefficient $C_{\mathrm{e}}$ is similar to the 
corresponding one for other substituted benzene compounds [11].

Since $\Delta v_{\mathrm{Q}}$ reflects the difference between the lattice contributions to each resonant site, namely $\mathrm{Cl}$ (I) and $\mathrm{Cl}$ (II), the change of $\Delta v_{\mathrm{Q}}$ may be associated with the onset of a neighbouring $\mathrm{OH}$ molecular group motion. Two temperature zones are distinguished for the data, i.e. $T<240$ and $T>240$, as may be seen from Figure 2. For each zone the data are best fitted by means of a second degree polynomial:

$$
\begin{gathered}
\Delta v^{\mathrm{I}}(\mathrm{kHz})=247.9-0.025 T-1.60 \cdot 10^{-4} T^{2}, \quad(6 \\
T<240 \mathrm{~K} . \\
\Delta v^{\mathrm{II}}(\mathrm{kHz})=271.8-0.247 T+3.53 \cdot 10^{-4} T^{2}, \\
T>240 \mathrm{~K} .
\end{gathered}
$$

Taking the difference between the $\Delta v_{\mathrm{Q}}$ data, for $T>240$, and the analytical fit corresponding to the low temperature zone, and plotting the logarithm of this difference vs. 1/T (insert in Fig. 2), a linear behaviour is found. The slope of the plotted difference $\delta v$ is $E_{\mathrm{d}}=(23.3 \pm 1.7) \mathrm{kJ} \mathrm{mol}^{-1}$, which shows that the contribution to $v_{\mathrm{Q}}$ by the $\mathrm{OH}$ group is given by

$$
\delta v^{\mathrm{OH}}=A+C e^{-E_{\mathrm{d}} / k T} .
$$

Although this equation is obtained by means of the above mentioned data manipulation, presently there are no basic principles available from which to deduce such a temperature behaviour of $\delta v^{\mathrm{OH}}$. Also, the temperature derivatives for both lines clearly depict the existence of two temperature intervals evidencing the two different lattice contributions to the EFG, Figure 3. The obtained value for $E_{\mathrm{d}}$ is close to the activation energy determined from the $T_{1}(T)$ data.

\section{Spin-Lattice Relaxation Time}

Spin lattice relaxation times were measured in the temperature range 100 to $310 \mathrm{~K}$ for each line, and depicted in Figure 4. From the data two temperature zones are clearly distinguished, i.e. $T<240 \mathrm{~K}$ and $T>240 \mathrm{~K}$. In the low temperature zone, $T_{1}$ behaves as $T_{1}=A T^{-\lambda}$ with $\lambda \cong 2.2$, indicating a relaxation mechanism mainly governed by torsional oscillations [12]. In the high temperature zone, $T_{1}(T)$ decreases faster than for low temperatures, also the rate of decrease of $v^{-}$is larger. This is

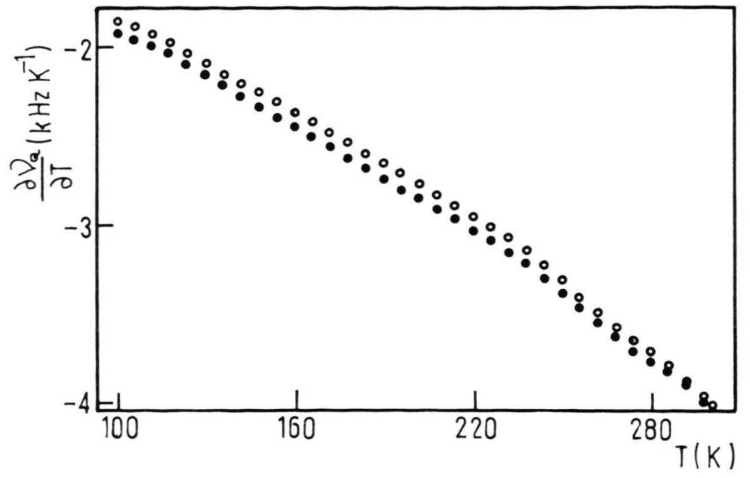

Fig. 3. Temperature derivatives $\left(\partial v_{\mathrm{Q}} / \partial T\right)$ for $v^{+}$(open circles) and $v^{-}$(solid circles).

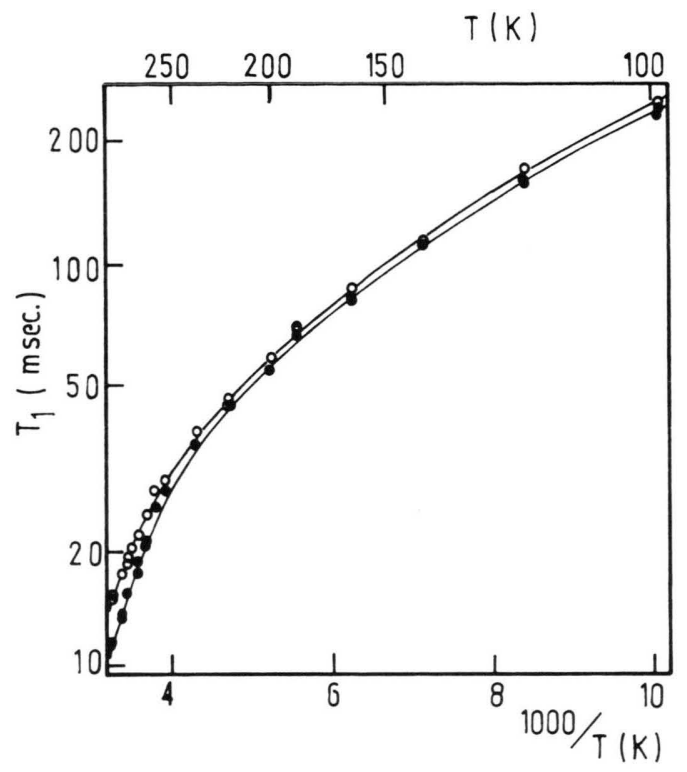

Fig. 4. Spin-lattice relaxation time for $v^{+}$(open circles) and $v^{-}$(solid circles). Solid lines correspond to fit with Equation (9).

associated with a reorientational motion of the $\mathrm{OH}$ group belonging to a neighbouring molecule. The change in the contribution to the EFG created at the resonant site by reorientational motion of a neighbouring atomic group should modulate the EFG at this nucleus and give rise to an effective spin-lattice relaxation mechanism with a rate [12]

$$
\left(\frac{1}{T_{1}}\right)_{\bmod }=\frac{1}{12}\left(\frac{e^{2} Q q^{\prime}}{\hbar}\right)^{2} \frac{\tau_{\mathrm{c}}}{1+\omega_{\mathrm{Q}}^{2} \tau_{\mathrm{c}}^{2}},
$$


where $q^{\prime}$ is that part of the EFG at the nucleus which changes with the reorientational motion and whose correlation time is

$$
\tau_{\mathrm{c}}=\tau_{0} e^{E_{\mathrm{a}} / k T},
$$

where $E_{\mathrm{a}}$ is the potential barrier hindering reorientation. The $T_{1}(T)$ data were fitted by means of

$\left(\frac{1}{T_{1}}\right)=\left(\frac{1}{T_{1}}\right)_{\mathrm{libr}}+\left(\frac{1}{T_{1}}\right)_{\bmod }=a T^{\lambda}+B e^{-E_{\mathrm{a}} / k T}$,

where the exponential in (9) is obtained from (7) when slow reorientational motion is assumed, i.e. $\omega_{\mathrm{Q}} \tau_{\mathrm{c}} \gg 1$. The fitting for both lines gives

$$
\lambda=2.24 \pm 0.02, \quad E_{\mathrm{a}}=(26.2 \pm 1.3) \mathrm{kJ} \mathrm{mol}^{-1} .
$$

A similar behaviour of $T_{1}$ as that given by (7) could be obtained by modulations of the dipole-dipole

[1] C. Dean and R. V. Pound, J. Chem. Phys. 20, 195 (1952).

[2] G. E. Peterson and P. M. Bridenbaugh, J. Chem. Phys. 46, 2644 (1967).

[3] P. Bucci, P. Cecchi, and A. Colligiani, J. Chem. Phys. 50, 530 (1969)

[4] M. Perrin and P. Michel, Acta Cryst. B 29, 253 (1973).

[5] H. Bayer, Z. Phys. 130, 227 (1951).

[6] T. Kushida, J. Sci. Hiroshima Univ. A 19, 327 (1955).

[7] G. Varsanyi, Assignments for Vibrational Spectra of Seven Hundred Benzene Derivates, Vol. 1, Adam Hilger, London 1974. interaction between the $\mathrm{Cl}$ and the $\mathrm{H}$ nuclei. However, such a possibility is discarded since the observed $T_{1}$ 's yield $\mathrm{Cl}-\mathrm{H}$ distances of the order of $0.1 \AA$. Besides, the change of curvature of $\Delta v_{\mathrm{Q}}$ at $240 \mathrm{~K}$ corroborates the above conclusion.

The parameter $B$ from (9) allows to determine the ratio of the $\mathrm{OH}$ contributions to $v_{\mathrm{Q}}$

$$
\frac{B^{(+)}}{B^{(-)}}=\left(\frac{v_{\mathrm{Q}}^{-}}{v_{\mathrm{Q}}^{+}}\right)^{2}\left|\frac{\delta v_{(+)}^{\mathrm{OH}}}{\delta v_{(-)}^{\mathrm{OH}}}\right|^{2}, \quad \delta v_{(-)}^{\mathrm{OH}}=1.4 \delta v_{(+)}^{\mathrm{OH}}
$$

which clearly shows that the $\mathrm{OH}$ group contributes more to the resonant $\mathrm{Cl}$ in type I molecules, giving rise to $v^{-}$. This is also plausible since type I molecules are arranged in loose dimers, and it may be this which causes a lowering of the $\mathrm{Cl}$ (I) NQR frequency relative to $\mathrm{Cl}$ (II) [13].

[8] G. P. O'Leary, Phys. Rev. Lett. 23, 782 (1969).

[9] R. J. C. Brown, J. Chem. Phys. 32, 116 (1960).

[10] N. Le Calve, M. H. Limage, S. Parent, and B. Pasquier, J. Chim. Phys. 74, 917 (1977).

[11] R. Righini and S. Califano, J. Raman Spectrosc. 7, 18 (1978).

[12] D. E. Woessner and H. S. Gutowsky, J. Chem. Phys. 39, 440 (1963).

[13] R. J. C. Brown, D. C. Woodland, and R. de Boer, J. Faraday Trans. II, 75, 1050 (1979). 